\title{
Quantum Mechanical Explanation for Dark Energy, Cosmic Coincidence, Flatness, Age, and Size of the Universe
}

https://doi.org/10.1515/astro-2019-0021

Received Sep 27, 2019; accepted Dec 16, 2019

\begin{abstract}
One of the most important problems in astronomy is the cosmological constant problem in which conventional calculation of vacuum energy density using quantum mechanics leads to a value which is $\sim 10^{123}$ times more than the vacuum energy estimated from astronomical observations of expanding universe. The cosmic coincidence problem questions why matter energy density is of the same order of magnitude as the vacuum energy density at present time. Finally, the mechanism responsible for spatial flatness is not clearly understood. In this paper, by taking the vacuum as a finite and closed quantum oscillator, we solve all of the above-mentioned problems. At first, by using the purely quantum mechanical approach, we predict that the dark energy density is $c^{4} /\left(G R^{2}\right)=5.27 \times 10^{-10} \mathrm{~J} / \mathrm{m}^{3}$ (where $R$ is radius of 3-sphere of the universe) and matter energy density is $c^{4} /\left(2 G R^{2}\right)=2.6 \times 10^{-10} \mathrm{~J} / \mathrm{m}^{3}$ which match well with astronomical observations. We also prove that dark energy has always been $\sim 66.7 \%$ and matter energy has been $\sim 33.3 \%$ of the total energy and thus solve the cosmic coincidence problem. Next, we show how flatness of space could be maintained since the early stage of the universe. Finally, using our model, we derive the expression for age and radius of the universe which match well with the astronomical data.
\end{abstract}

Keywords: dark energy, cosmological constant problem, cosmic coincidence problem, flatness problem, Hubble's law, age of the universe, radius of the universe

\section{Introduction}

General theory of relativity and quantum mechanics (or quantum field theory) developed in last century are the two pillars on which the whole of modern physics now rests. Independently each of them has proved itself to be correct with surprising accuracy. For instance, bending of light coming from distant stars by the Sun and precession of perihelion of Mercury predicted by general relativity have been exactly reproduced in astronomical observations. Similarly, prediction of value of fine structure constant by quantum electrodynamics has been tested with a precision of about one part in billion. So, it is natural to expect both these jewels of physics to converge when calculating some common parameters of the universe. But, surprisingly, predictions of both these theories fall apart by unexpected amounts while calculating some of the important astro-

\footnotetext{
Corresponding Author: Biswaranjan Dikshit: Laser and Plasma Technology Division, Bhabha Atomic Research Centre, Mumbai400085, INDIA and Homi Bhabha National Institute, Mumbai, India; Email: bdikshit73@yahoo.co.in
}

๑ Open Access. ๑ 2019 B. Dikshit, published by De Gruyter License nomical parameters such as dark energy (or vacuum energy) responsible for accelerated expansion of the universe. This is known as cosmological constant problem. Of course, there exists some modified gravity theories (Yousaf et al. 2016a,b; Yousaf 2017, 2018, 2019a; Yousaf et al. 2019b)) such as $f(R, T)$ theory (where $R$ is Ricci scalar and $T$ is trace of energy-momentum tensor) which can explain the gravitational collapse, structure formation and the expansion of the universe without bringing in dark energy. But, in this paper, we will not follow that approach as the nature of function $f(R, T)$ is arbitrarily assumed and we are in search of a quantum mechanical basis for astronomical observations. In other questions related to ratio of matter energy to dark energy, flatness of space, present age and size of universe, presently quantum theory is unable to provide any satisfactory explanation and in this article we will attempt to solve all these problems. Let us first discuss these problems in detail and then progress to the proposed solutions of these in next sections.

First comes the cosmological constant problem. The original form of Einstein's field equation which determines 
the space-time structure is given by,

$$
G_{\mu \nu}=\frac{8 \pi G}{c^{4}} T_{\mu \nu}
$$

Where $G_{\mu \nu}$ is Einstein tensor, $T_{\mu \nu}$ is energy-momentum tensor, $G$ is Newton's gravitational constant and $\boldsymbol{c}$ is speed of light.

When Einstein (1917) applied above equation to a static, isotropic and homogenous universe in the form of 3-sphere containing only matter (pressure less dust), he found that Eq. (1) is not satisfied. So, to solve the problem, he added a cosmological constant $\Lambda$ at the left-hand side of Eq. (1) as given below.

$$
G_{\mu \nu}+\frac{\Lambda}{c^{2}} g_{\mu \nu}=\frac{8 \pi G}{c^{4}} T_{\mu \nu}
$$

where $g_{\mu \nu}$ is metric tensor with local space-time signature $(-,+,+,+)$. We have to note that the constant $\Lambda$ had been artificially added by Einstein just to make the universe static. Later on, Friedmann (1922) developed a model for an expanding universe using Einstein's field equation given by Eq. (2) that includes the cosmological constant $\Lambda$. The Friedmann's equations are (Carroll 2001),

$$
\begin{aligned}
& \left(\frac{\dot{R}}{R}\right)^{2}=\frac{8 \pi G}{3} \rho_{\text {nonvac }}+\frac{\Lambda}{3} c^{2}-\frac{k}{R^{2}} c^{2} \\
& \frac{\ddot{R}}{R}=-\frac{4 \pi G}{3}\left(\rho_{\text {nonvac }}+3 \frac{p}{c^{2}}\right)+\frac{\Lambda}{3} c^{2}
\end{aligned}
$$

Where ' $R$ ' is the scale factor in Friedmann-RobertsonWalker (FRW) metric and physically it is the radius of the universe in the form of 3-sphere expanding in 4dimensional space. $k$ is curvature constant which can be +1 (for closed universe), 0 (for flat universe) or -1 (for open universe), $\rho_{\text {nonvac }}$ is density of non-vacuum component (ordinary matter, dark matter and radiation).

If we write $\Lambda=\frac{8 \pi G}{c^{2}} \rho_{v a c}$, Eq. (3) becomes,

$$
\left(\frac{\dot{R}}{R}\right)^{2}=\frac{8 \pi G}{3}\left(\rho_{\text {nonvac }}+\rho_{\text {vac }}\right)-\frac{k}{R^{2}} c^{2}
$$

Using the redshift-brightness data of type Ia supernovae in distant galaxies which act as standard candles, we can measure the ratio of velocity of recession of galaxies to their distance from us which is known as $\left(\frac{\dot{R}}{R}\right)=H$ (Hubble's constant). Taking observed data from (Planck Collaboration 2016), $H \approx 67.9 \mathrm{~km} \mathrm{~s}^{-1} \mathrm{Mpc}^{-1}$ and $\rho_{\text {nonvac }} \approx 0.31 \rho_{\text {crit }}$ where critical density $\rho_{\text {crit }}=\frac{3 H^{2}}{8 \pi G}$. Putting $k=0$ (since universe is found to be flat), we can calculate the vacuum energy density $\rho_{v a c} c^{2}$ from Eq. (5) and its value is found to be $\sim 5.4 \times 10^{-10} \mathrm{~J} / \mathrm{m}^{3}$. This is also called dark energy density.

Qualitatively, the idea of vacuum energy in general relativity is very well compatible with quantum field theory which considers the free space to have zero-point or ground state energy. In fact, the existence of zero-point energy has been experimentally demonstrated through Lamb shift of atomic spectra, modification of magnetic moment of electron and Casimir effect (Casimir 1948; Mohideen 1998; Lamoreaux 1997; Wilson et al. 2011) which causes an attractive force between two closely separated conducting bodies. However, when we go for a quantitative comparison, a huge surprise appears before us. Considering all possible modes of zero-point oscillation, as shown by Weinberg (1989), quantum mechanical vacuum zero-point energy density is given by $\sim \frac{\pi^{2} \hbar c}{l_{p}^{4}}$, where $l_{p}$ is the minimum possible wavelength taken to be Planck length. By putting the values of constants, this quantum vacuum zero-point energy density comes out to be $\sim 10^{114} \mathrm{~J} / \mathrm{m}^{3}$ which is about $10^{123}$ times more than the value estimated from astronomical observations. This is the worst theoretical predication in the history of modern physics which is commonly known as cosmological constant problem (Weinberg 1989; Banks 2004; Peebles 2003).

In an effort to explain the astronomically observed small value of vacuum energy, few theories have been proposed in literature which certainly are not convincing. Details of these theories and their loop holes are described by Weinberg (1989). For example, supersymmetry assumes that corresponding to each fermion, there exists a boson resulting in cancellation of energy. But how can this cancellation be so precise that vacuum energy density comes down from $\sim 10^{114} \mathrm{~J} / \mathrm{m}^{3}$ to $\sim 5.4 \times 10^{-10} \mathrm{~J} / \mathrm{m}^{3}$ ? In addition, till date no supersymmetric particle has been detected experimentally. Another important proposal is the anthropic principle. In this principle, it is stated that even if cosmological constant can take any random values, we will observe it to be as it is today since it is the only value that allows formation of galaxies and stars by gravitational condensation and creation of intelligent life who can measure it. But in my view, our existence can be an effect of present state of the universe, it cannot be the cause. In another approach to explain the present low value of vacuum energy density, Chen and Wu (1990) have postulated cosmological constant to be varying as $R^{-2}$ where $R$ is scale factor of universe. But, they have not given any scientific justification for such a variation. Similarly, many other researchers (Berman 1991; Carvalho et al. 1992; Fujii and Nishioka 1990; Rubakov 2000) have also assumed relaxation of cosmological constant with time, that too again without sufficient reason. They have just analyzed the consequences of such a variation. Understanding the origin of dark energy becomes more important considering the recent work of (Yousaf 2019a; Yousaf et al. 2019b) on gravastar (gravitational vacuum star) using modified gravity. Gravastar is a 
(hypothesized) celestial body that has a central vacuum core containing dark energy which produces a repulsive force and thus avoids formation of singular point.

Next unanswered question in Astrophysics is the problem of cosmic coincidence (Velten et al. 2014). Although matter density decreases as $1 / r^{3}$ in an expanding universe of radius $r$ (from nearly $\infty$ to zero) and vacuum energy density is believed to be constant, it is a surprising coincidence that both are of same order at the present time. Energy in the form of matter and radiation (including dark matter) is estimated to be $\sim 32 \%$ and the rest $\sim 68 \%$ is in the form of vacuum energy. Zlatev et al. (1999) had invoked the concept of an additional "tracker field" which roles down a potential to keep ratio of dark energy to matter energy at present value. However, in contrast to vacuum energy whose effects have been physically observed (in form of Lamb shift, Casimir effect etc), effect of this tracker field has never been experimentally observed. So, as correctly commented by Velten et al. (2014), this solution seems artificial.

Next important question in cosmology is flatness problem of space. Space is found to be flat $(k=0)$ to the best of our observational accuracy. This is possible only if the total energy density of the space including matter/radiation becomes exactly equal to the critical density $\left(\rho_{c r i t}=\frac{3 H^{2}}{8 \pi G}\right)$. As estimated by Guth (1981), the density of universe must be exactly equal to the critical density with a precision of about one part in $10^{55}$ since the early stage of the universe to till date. If it were not so, the universe would have either re-collapsed to a point as soon as it was born (in case of slightly higher density) or would have expanded at such a large rate that galaxies and stars would not have been formed by condensation (in case of slightly lower density). To some extent, Guth $(1981,1997)$ has explained this problem by his cosmic inflation theory which assumes a specific type of potential for false vacuum represented by a scalar field (inflaton). But, it is not clear what caused this particular type of potential for the scalar field.

In this paper, by adopting a purely quantum mechanical approach, we have calculated the vacuum or dark energy density which matches well with the astronomically observed value. Thus, the earlier disagreement by a factor of $\sim 10^{123}$ between quantum field theory and cosmology disappears. Then, combining the law of thermodynamics with this result, we have estimated the total non-vacuum (matter plus radiation) energy density of universe which is again found to be nearly the same as the observed data. We have also proved that the ratio of dark energy to total energy and the ratio of matter energy to total energy have been of same order i.e. $\sim 2 / 3$ and $\sim 1 / 3$ respectively since the early stage of universe. Thus, the so called cosmic coincidence is not a coincidence at all. Then, using our expressions of energy density in Friedmann equations, we have explained how flatness of space has been maintained since the early stage of universe. Subsequently, we mathematically prove that the Hubble's constant is exactly equal to the reciprocal of age of universe. Thus, we estimate the present age and radius of universe which match well with the astronomical data.

\section{Quantum mechanical calculation of dark energy density (Solution of cosmological constant problem)}

Let us calculate the vacuum (or dark) energy density of free space of the universe. As Einstein (1917) and Friedmann (1922) had visualized, we will assume the universe to be an isotropic, homogenous and closed 3-sphere. So, the 3-D space of the universe is actually the surface of a 3-sphere existing in a four-dimensional space. All matter and radiation are bound to this surface and the fourth dimension along radial direction is not observable. This is due to the fact that, as per quantum field theory, all matter and radiation are truly waves (Hobson 2013) and all these waves must lie on the surface of 3-sphere. Just like the vibration of a string cannot leave the string, no object (or wave packet) can leave the surface of 3-sphere to move along fourth dimension. Space itself acts as the quantum oscillator which shows the wave behavior. So, naturally it must have the zero-point (or ground state) energy. Sum of zero-point energies for all possible modes of oscillations is known as vacuum energy.

Since, it is the quantum oscillator which is responsible for energy, we will assume that zero-point oscillator for all the fundamental particles (electron, proton, photon, neutrino, quark etc.) is the same. Properties of particles other than energy appear when this oscillator in excited state couples with other fields such as Higg's field (giving rise to mass), electromagnetic field (giving rise to charge), etc... So, although there are 17 different fundamental particles, we will consider only a single and common zero-point oscillation in space for all particles of same energy.

It is well known that for a particle-wave trapped in a three-dimensional box having boundaries, three different quantization conditions appear i.e. each of length ' $a$ ', breadth ' $b$ ' and height ' $c$ ' must be an integer multiple of half wave length corresponding to the momentum along 
that direction $\left(a=n_{x} \frac{h}{2 p_{x}}, \quad b=n_{y} \frac{h}{2 p_{y}}, \quad c=n_{z} \frac{h}{2 p_{z}}\right)$. But in a boundary-less situation having spherical symmetry such as Bohr's model of hydrogen atom, only one quantization condition appears i.e. circumference must be integer multiple of wavelength corresponding to total momentum, $2 \pi r=n \lambda=n \frac{h}{p} \Rightarrow L=p r=n \hbar$. Thus, Bohr reached at the quantized value of angular momentum of electron. Similarly, as the space of our universe is boundary-less (closed) and spherically symmetric, we will have only one quantization condition for the zero-point oscillation. So, if ' $R$ ' is the radius of universe in the form of 3-sphere existing in four-dimensional space and ' $\lambda$ ' is wavelength of zero-point oscillation, we get,

$$
2 \pi R=n \lambda
$$

where $n$ is an integer varying from 1 to $n_{\max }$ and the value of $n_{\max }$ is decided by lowest possible value of wavelength $\lambda_{\min }$.

We know when the wave length is of the order of Planck length $l_{p}=\sqrt{\frac{\hbar G}{c^{3}}}\left(=1.6 \times 10^{-35} \mathrm{~m}\right)$, it modifies the metric of space around itself to such an extent that it becomes a tiny black hole (Saslow 1998; Lattman 2009). So, roughly speaking, wavelength smaller than Planck length cannot exist. In this paper, we will take the cutoff wavelength as $\lambda_{\min }=\frac{l_{p}}{\sqrt{2}}$ for which a justification can be given as follows. For the zero-point oscillation which is a standing wave in closed universe, the momentum can be $+p$ or $-p$. Thus, momentum uncertainty (standard deviation) is, $\Delta p=p$. Then, uncertainty in position is,

$$
\Delta x \geq \frac{\hbar}{2 p}
$$

This position uncertainty must also be more than Schwarzschild radius $r_{s}$. Mathematically,

$$
\Delta x \geq r_{s}
$$

Or

$$
\Delta x \geq \frac{2 G E}{c^{4}}
$$

( $E$ is energy of particle)

As for zero-point oscillation, $E=\frac{1}{2} h v=\frac{h c}{2 \lambda}=\frac{p c}{2}$. So we get,

$$
\Delta x \geq \frac{G p}{c^{3}}
$$

Multiplying Eq. (7) and (8),

$$
(\Delta x)^{2} \geq \frac{G \hbar}{2 c^{3}}
$$

Or

$$
\Delta x \geq \frac{l_{p}}{\sqrt{2}}
$$

where $l_{p}=\sqrt{\frac{G \hbar}{c^{3}}}=$ Planck length

Since we have found that position uncertainty of zeropoint oscillators is always more than $\frac{l_{p}}{\sqrt{2}}$, wavelength less than this value cannot be defined and minimum possible wavelength can be taken as, $\lambda_{\min }=\frac{l_{p}}{\sqrt{2}}$.

Using the above minimum wavelength in Eq. (6) we get,

$$
n_{\max }=\frac{2 \pi R}{\lambda_{\min }}=\frac{2 \sqrt{2} \pi R}{l_{p}}
$$

Taking zero-point wave as mass-less (doesn't couple with Higg's field), its velocity will be the same as the speed of light. So, its frequency will be, $v=c / \lambda$. Now, the total vacuum energy of the universe due to zero-point oscillations is,

$$
U_{v a c}=\sum \frac{1}{2} h v=\frac{h c}{2} \sum \frac{1}{\lambda}
$$

Using Eq. (6),

$$
U_{v a c}=\frac{h c}{4 \pi R} \sum_{1}^{n_{\max }} n=\frac{h c}{4 \pi R} \frac{n_{\max }\left(n_{\max }+1\right)}{2}
$$

Or

$$
U_{v a c}=\frac{\hbar c}{4 R} n_{\max }^{2}\left(1+\frac{1}{n_{\max }}\right)
$$

Putting the value of $n_{\max }$ from Eq. (10) in above expression,

$$
U_{v a c}=\frac{\hbar c}{4 R}\left(\frac{2 \sqrt{2} \pi R}{l_{p}}\right)^{2}\left(1+\frac{l_{p}}{2 \sqrt{2} \pi R}\right)
$$

Or

$$
U_{v a c}=\frac{2 \pi^{2} \hbar c R}{l_{p}^{2}}\left(1+\frac{l_{p}}{2 \sqrt{2} \pi R}\right)
$$

Putting the definition of Plank length, $l_{p}=\sqrt{\frac{\hbar G}{c^{3}}}$, total vacuum energy becomes,

$$
U_{v a c}=\frac{2 \pi^{2} c^{4} R}{G}\left(1+\frac{l_{p}}{2 \sqrt{2} \pi R}\right)
$$

Since the total volume of space $=$ surface area of 3-sphere $=2 \pi^{2} R^{3}$, dividing Eq. (13) by it, we get the vacuum energy density (in $\mathrm{J} / \mathrm{m}^{3}$ ) as,

$$
u_{v a c}=\frac{U_{v a c}}{2 \pi^{2} R^{3}}
$$

Or

$$
u_{v a c}=\rho_{v a c} c^{2}=\frac{c^{4}}{G R^{2}}\left(1+\frac{l_{p}}{2 \sqrt{2} \pi R}\right)
$$

As $R \gg l_{p}$ (after early universe), second term in Eq. (13) and (14) becomes negligible. So, total vacuum energy and vacuum energy density (in $\mathrm{J} / \mathrm{m}^{3}$ ) are given by,

$$
U_{v a c}=\frac{2 \pi^{2} c^{4} R}{G}
$$




$$
u_{v a c}=\rho_{v a c} c^{2}=\frac{c^{4}}{G R^{2}}
$$

Or mass density of vacuum is,

$$
\rho_{v a c}=\frac{c^{2}}{G R^{2}}
$$

Thus, from Eq. (15) and (16), we find that total vacuum energy increases linearly with radius of universe $R$ along $4^{\text {th }}$ dimension and vacuum energy density is inversely proportional to $R^{2}$. It is interesting to note that although we had started from purely quantum mechanical approach, its representative constant $\hbar$ has disappeared from the final expression of vacuum energy density in Eq. (16) migrating us from quantum physics (physics of small) to general relativity (physics of large). Putting the values of constants $c$, $G$ and radius of universe as $R=4.8 \times 10^{26} \mathrm{~m}$ (we will prove it in section 5), we get the vacuum energy density $u_{v a c}$ as $5.27 \times 10^{-10} \mathrm{~J} / \mathrm{m}^{3}$ which matches well with the astronomical observed value $\sim 5.4 \times 10^{-10} \mathrm{~J} / \mathrm{m}^{3}$. Thus, we have now closed the earlier gap of the order of $\sim 10^{123}$ between quantum physics and cosmology and the cosmological constant problem is solved.

\section{Solution of Cosmic coincidence problem}

Let the internal energy due to non-vacuum components (ordinary matter, dark matter and radiation) of the universe is $U_{\text {nonvac }}$ and the corresponding energy density is $u_{\text {nonvac }}$. Assuming expansion of the universe is an adiabatic process, first law of thermodynamics dictates,

$$
d\left(U_{v a c}+U_{\text {nonvac }}\right)+p d V=0
$$

Where $p$ is the pressure and $V$ is volume.

Pressure due to non-vacuum component is negligible as speed of matter (stars and galaxies) is much less than speed of light and amount of radiation energy is less than $10^{-3} \%$. So, pressure is caused by vacuum. Conventionally in cosmology, vacuum is thought to be a perfect fluid with negative pressure given by, $p=-\rho_{v a c} c^{2}$. But in our case, vacuum is considered to be a zero-point harmonic oscillator which carries its half energy in the form of potential energy and another half in the form of kinetic energy. Only the potential energy part in our case can cause pressure, whereas kinetic part cannot as there is no change in momentum of zero-point oscillator (for example, in a box, a particle having kinetic energy causes pressure on wall only when it's momentum changes by collision with wall). So, pressure due to vacuum in our case becomes,

$$
p=-\frac{1}{2} \rho_{v a c} c^{2}
$$

So,

$$
p d V=-\frac{1}{2} \rho_{v a c} c^{2} d V
$$

Putting the value of $\rho_{v a c}$ from Eq. (14) in above and as $V=$ $2 \pi^{2} R^{3}$,

$$
p d V=-\frac{1}{2} \frac{c^{4}}{G R^{2}}\left(1+\frac{l_{p}}{2 \sqrt{2} \pi R}\right) 2 \pi^{2} d\left(R^{3}\right)
$$

Or

$$
p d V=-\frac{3 \pi^{2} c^{4}}{G}\left(1+\frac{l_{p}}{2 \sqrt{2} \pi R}\right)
$$

Note that work done by the system is negative implying that its internal energy increases during expansion.

Removing the bracket in Eq. (13), total vacuum energy is,

$$
U_{v a c}=\frac{2 \pi^{2} c^{4} R}{G}+\frac{\pi c^{4} l_{p}}{\sqrt{2} G}
$$

Taking differential on both sides of above equation, we get,

$$
d U_{v a c}=\frac{2 \pi^{2} c^{4}}{G} d R
$$

Putting Eq. (20) and (21) in Eq. (18), we get,

$$
d U_{\text {nonvac }}=-\frac{2 \pi^{2} c^{4}}{G} d R+\frac{3 \pi^{2} c^{4}}{G}\left(1+\frac{l_{p}}{2 \sqrt{2} \pi R}\right) d R
$$

Or

$$
d U_{\text {nonvac }}=\frac{\pi^{2} c^{4}}{G}\left(1+\frac{3 l_{p}}{2 \sqrt{2} \pi R}\right) d R
$$

Thus, we find that non-vacuum energy of the universe also increases with radius of the universe and it is being created by the expansion of space. Increase of non-vacuum energy (i.e. creation of matter and radiation) happens by way of excitation of zero-point oscillators. Integrating Eq. (22), we get,

$$
U_{\text {nonvac }}=\frac{\pi^{2} c^{4}}{G}\left[R-R_{i}+\frac{3 l_{p}}{2 \sqrt{2} \pi} \ln \left(\frac{R}{R_{i}}\right)\right]
$$

Where $R_{i}$ is initial radius of universe. $R_{i}$ can be found from Eq. (10) by putting $n_{\max }$ equal to 1 and it is given by, $R_{i}=$ $\frac{l_{p}}{2 \sqrt{2} \pi}=1.81 \times 10^{-36} \mathrm{~m}$. Thus, Eq. (23) becomes,

$$
U_{\text {nonvac }}=\frac{\pi^{2} c^{4} R}{G}\left[1-\frac{R_{i}}{R}+\frac{3 R_{i}}{R} \ln \left(\frac{R}{R_{i}}\right)\right]
$$


Dividing Eq. (24) by volume $\left(2 \pi^{2} R^{3}\right)$, we get energy density due to non-vacuum component given by,

$$
\begin{aligned}
u_{\text {nonvac }} & =\rho_{\text {nonvac }} c^{2} \\
& =\frac{c^{4}}{2 G R^{2}}\left[1-\frac{R_{i}}{R}+\frac{3 R_{i}}{R} \ln \left(\frac{R}{R_{i}}\right)\right]
\end{aligned}
$$

Just at the time of creation of universe, when $R=R_{i}$, Eq. (24) and (25) indicate that the non-vacuum energy (matter/radiation) and its density were zero. The non-vacuum energy density initially increased with $R$, became maximum when $\frac{d u_{\text {nonvac }}}{d R}=0$ and then decreased with $R$. So, taking the derivative of Eq. (25) equal to zero and then numerically solving it, we can get the radius of universe $\left(R_{\max \_d e n s i t y}\right)$ at which maximum density must have occurred. That comes out to be,

$R_{\text {max _density }}=1.42 \times R_{i}=1.42 \times \frac{l_{p}}{2 \sqrt{2} \pi}=2.57 \times 10^{-36} \mathrm{~m}$

And corresponding energy density comes out to be $\left(\right.$ unonvac $_{\max }=9.5 \times 10^{114}$ joule $/ \mathrm{m}^{3}$.

However, as soon as the radius of universe became much more than initial radius or Planck length $\left(\sim 10^{-35} \mathrm{~m}\right)$ i.e. $R \gg R_{i}$, Eq. (24) and (25) reduce to

$$
\begin{gathered}
U_{\text {nonvac }}=\frac{\pi^{2} c^{4} R}{G} \\
u_{\text {nonvac }}=\rho_{\text {nonvac }} c^{2}=\frac{c^{4}}{2 G R^{2}}
\end{gathered}
$$

In the units of $\mathrm{kg} / \mathrm{m}^{3}$, the non-vacuum mass density is,

$$
\rho_{\text {nonvac }}=\frac{c^{2}}{2 G R^{2}}
$$

Comparing Eq. (27) and (16), we find that the ratio of nonvacuum energy to the vacuum energy is $\sim 1 / 2$ and it is independent of the radius $\mathrm{R}$ of universe (when it is $\gg 10^{-35} \mathrm{~m}$ ). In other words, theoretically, $\sim 66.7 \%$ of total energy should be in the form of dark energy and the rest $33.3 \%$ should be in the form of non-vacuum component i.e. matter (including dark matter) and radiation since the beginning of universe. So, cosmic coincidence problem is not really there as the proportion of different components of energy has been a constant and will remain so forever.

The absolute value of predicted non-vacuum energy density calculated from Eq. (27) comes out to be $\sim 2.6 \times 10^{-10}$ $\mathrm{J} / \mathrm{m}^{3}$ and astronomically observed value (Planck Collaboration 2016) is $\sim 2.4 \times 10^{-10} \mathrm{~J} / \mathrm{m}^{3}$. The reason for astronomically observed density of matter being slightly lower than theoretical value may be due to the fact that we have some inaccuracies in estimating the amount of dark matter in distant galaxies.
It may be noted that total energy of universe can be calculated by adding Eq. (15) and (26) which comes out to be, $U_{\text {Total }}=U_{\text {vac }}+U_{\text {nonvac }}=\frac{3 \pi^{2} c^{4} R}{G}$. So, in an expanding universe, the total energy increases with $R$ i.e. radius along $4^{\text {th }}$ dimension of space. Then, where does this energy come from? This energy comes from the four-dimensional space in the form of work ( $P d V$ ) (see Eq. (20)) during expansion of three-dimensional vacuum having negative pressure. This is opposite to the phenomena like expansion of a gas with positive pressure in a cylinder-piston system losing energy in the form of work to its environment i.e. piston. Since in our case, vacuum has negative pressure, its expansion causes gain of energy in the form of work from its environment i.e. four-dimensional space. At the instant of creation of universe, Eq. (11) and (24) indicate that total energy of universe was purely dark energy (non-vacuum part was zero) and its value was $\sim 8.7 \times 10^{9} \mathrm{~J}$ which might have been created from quantum fluctuation.

\section{Solution of Flatness problem}

As stated earlier, Einstein had artificially introduced the cosmological constant $\Lambda$ in Eq. (2) just to make the universe static. However, when the observations indicated an expanding universe, he regretted to adding it. So, in this paper we will use the original version of Einstein field equation given by Eq. (1), but we will include the vacuum energy contribution in the energy-momentum tensor. So, in our case, Eq. (1) will be,

$$
G_{\mu \nu}=\frac{8 \pi G}{c^{4}} T_{\mu \nu}^{t o t a l}
$$

Friedmann's solution of above equation will be,

$$
\begin{aligned}
& \left(\frac{\dot{R}}{R}\right)^{2}=\frac{8 \pi G}{3} \rho^{\text {total }}-\frac{k}{R^{2}} c^{2} \\
& \frac{\ddot{R}}{R}=-\frac{4 \pi G}{3}\left(\rho^{\text {total }}+3 \frac{p}{c^{2}}\right)
\end{aligned}
$$

Writing $\rho^{\text {total }}=\rho_{\text {vac }}+\rho_{\text {nonvac }}$ in the above equation and substituting quantum mechanical expressions for $\rho_{v a c}$, $\rho_{\text {nonvac }}$ and $p$ from Eq. (17), (28) and (19) respectively, we get,

$$
\begin{aligned}
& \left(\frac{\dot{R}}{R}\right)^{2}=\frac{8 \pi G}{3}\left(\frac{c^{2}}{G R^{2}}+\frac{c^{2}}{2 G R^{2}}\right)-\frac{k}{R^{2}} c^{2} \\
& \ddot{R}=-\frac{4 \pi G}{3}\left(\frac{c^{2}}{G R^{2}}+\frac{c^{2}}{2 G R^{2}}-\frac{3}{2} \frac{c^{2}}{G R^{2}}\right)=0
\end{aligned}
$$


Simplifying the above, two Friedmann equations in our case become,

$$
k=4 \pi-\left(\frac{\dot{R}}{c}\right)^{2}
$$

And

$$
\dot{R}=\text { Constant }
$$

Thus, General relativity in the form of Friedmann's solution requires that Eq. (32) and (33) must be simultaneously satisfied. Since Eq. (33) needs $\dot{R}$ to be constant, putting it in Eq. (32), we get that $k$ is also a constant in time. Thus, general relativity when combined with quantum mechanics dictates that both $\dot{R}$ and $k$ are constants. When $R=\infty$, energy density will be zero (from Eq. (16) and (27)) and so space must be flat i.e. $k=0$. Since just now we have proved that $k$ is time independent, it must be zero as soon as $R \gg R_{i}\left(\sim 10^{-35} \mathrm{~m}\right)$. In other words, space must be flat or total density of universe must always be equal to critical density since the early stage of universe. Thus, flatness of space is achieved not by a mysterious fine tuning of density, but due to $1 / r^{2}$ dependence of both dark energy density and matter density as given by Eq. (16) and (27).

From Eq. (32), we get that for a flat space $(k=0)$,

$$
\dot{R}=2 \sqrt{\pi} c
$$

i.e. radius of universe increases at a constant speed of $2 \sqrt{\pi} c$ along the $4^{\text {th }}$ dimension. This cannot be taken as violating the special theory of relativity as we are here talking about speed of space in the form of a 3-sphere in 4-dimensional space whereas special relativity limits the velocity of particles in 3-dimensional space.

In fact, we can prove the flatness of space irrespective of the size of universe by a simpler procedure. We know, if the total density becomes equal to critical density $\left(\rho_{\text {crit }}=\frac{3 H^{2}}{8 \pi G}\right)$, then space becomes flat. Putting, $H=$ $\frac{\dot{R}}{R}$ and $\dot{R}=2 \sqrt{\pi} c$, the required critical density becomes, $\left(\rho_{\text {crit }}=\frac{3 c^{2}}{2 G R^{2}}\right)$. Now total density of universe can be calculated by adding vacuum and nonvacuum parts as given by Eq. (17) and (28) which comes out to be, $\left(\rho_{\text {total }}=\rho_{\text {vacuum }}+\rho_{\text {nonvacuum }}=\frac{3 c^{2}}{2 G R^{2}}\right)$. Thus, total density is always equal to the critical density required for flatness of space whatever be the radius of universe. So, our model solves the flatness problem of universe.

\section{Estimation of age and radius of universe}

Let $\theta$ be the angular distance of a galaxy from us on 3-sphere (i.e. universe). Let $s$ and $\dot{s}$ be the relative distance and relative velocity of recession of the galaxy from us at present time $t_{0}$ when radius of universe is $R$. Since red shift occurs due to expansion of intermediate space between galaxy and us and the intensity of light also depends upon present spatial distance, measured values $s$ and $\dot{s}$ represent the relative distance and relative velocity of recession of galaxy at present time $t_{0}$. As $s=R \theta$ and angular distance $\theta$ remains constant irrespective of size of universe, we get,

$$
\frac{\dot{s}}{s}=\left.\frac{\dot{R}}{R}\right|_{t_{0}}
$$

Using Eq. (34) which dictates that radius of universe along the $4^{\text {th }}$ dimension increases at a constant speed of $2 \sqrt{\pi} c$, we get,

$$
\left.\frac{\dot{R}}{R}\right|_{t_{0}}=\frac{2 \sqrt{\pi} c}{2 \sqrt{\pi} c t_{0}}=\frac{1}{t_{0}} .
$$

Since in the present period of measurement, $t_{0}$ is approximately constant (measurements have been done during last century whereas present age of universe is few billion years), we can take $\left.\frac{\dot{R}}{R}\right|_{t_{0}}=\frac{1}{t_{0}}$ a constant which we call "Hubble's constant $\left(H_{0}\right)$ ", Thus,

$$
\left.\frac{\dot{R}}{R}\right|_{t_{0}}=\frac{1}{t_{0}}=H_{0}
$$

Putting the above expression in Eq. (35), we get,

$$
\dot{s}=H_{0} s \text { (Hubble's law) }
$$

Thus, we have proved that the constant in Hubble's law is exactly equal to the reciprocal of age of universe.

From Eq. (36), we can find the age of universe. Taking $H_{0}=67.9 \mathrm{~km} \mathrm{~s}^{-1} \mathrm{Mpc}^{-1}$ from (Planck Collaboration 2016), age of universe comes out to be $t_{0} \sim 14.4$ billion years. This value closely agrees with the age $\sim 14.5$ billion years estimated by other independent methods such as from oldest globular clusters (Chaboyer et al. 1996).

Present radius of universe can be calculated by putting the value of $\dot{R}$ from Eq. (34),

$$
R=\dot{R} t_{0}=2 \sqrt{\pi} c t_{0}=\frac{2 \sqrt{\pi} c}{H_{0}}
$$

Taking the observed value of $\sim 67.9 \mathrm{~km} \mathrm{~s}^{-1} \mathrm{Mpc}^{-1}$ for Hubble's constant from (Planck Collaboration 2016), present radius of universe calculated from Eq. (38) comes out to be $\sim 4.8 \times 10^{26} \mathrm{~m}$ (or 50 billion light years). This matches well with the approximate value $\sim 4.4 \times 10^{26} \mathrm{~m}$ reported by (Bars and Terning 2009). 


\section{Conclusion}

In this paper, by using purely quantum mechanical method, we have calculated the vacuum (or dark) energy density to be $\frac{c^{4}}{G R^{2}}=5.2 \times 10^{-10} \mathrm{~J} / \mathrm{m}^{3}$ which agrees well with the astronomically observed value. Thus, the earlier disagreement by a factor of $\sim 10^{123}$ between quantum field theory and cosmology has disappeared solving the cosmological constant problem. Then, combining the law of thermodynamics with this result, we have theoretically estimated the non-vacuum energy density (ordinary matter, dark matter and radiation) of the universe which comes out to be $\sim \frac{c^{4}}{2 G R^{2}}=2.6 \times 10^{-10} \mathrm{~J} / \mathrm{m}^{3}$. This value also matches well with the observed data. The amount of dark energy and matter energy expressed as a percentage of total energy are estimated to be $\sim 66.7 \%$ and $\sim 33.3 \%$ respectively and the proportions are proved to be maintained since the early stage of universe. So, we have showed that the so called cosmic coincidence is not a coincidence at all. The minor difference between theoretically predicted density of matter $\left(\sim 0.33 \rho_{\text {crit }}\right)$ and astronomically observed value $\left(\sim 0.31 \rho_{\text {crit }}\right)$ may be due to some observational error in estimating the amount of dark matter in distant galaxies. We hope this will be confirmed in future by more accurate observations. Then, using our results in Friedmann equations, we have solved the flatness problem of space. We have also found that the universe in the form of a 3-sphere is expanding at the rate of along the unobservable $4^{\text {th }}$ radial dimension. We have shown that the time varying Hubble's constant is exactly equal to the age of universe. Estimated age and radius of universe in our approach come out to be $\sim 14.4$ billion years and $\sim 50$ billion light years respectively which match well with the literature data.

Acknowledgment: Author is grateful to Mr. R K Rajawat of Beam Technology Development Group, Bhabha Atomic Research Centre for his encouragement and support during this research. Author is also thankful to Prof James D Stein of California State University for his thorough reading of the manuscript and useful feedbacks that improved the presentation of the paper.

\section{References}

Banks, T. 2004, Physics Today, 57(3), 46-51.

Bars, I. \& Terning, J. 2009, “Extra dimensions in Space and Time”, Springer, p. 27.

Berman, M. S. 1991, Phys. Rev. D, 43(4), 1075-1078.

Carroll, S. M. 2001, Living Rev. Relativity, 4(1), 1-56.

Carvalho, J. C., Lima, J. A. S., \& Waga, I. 1992, Phys. Rev. D, 46(6), 2404-2407.

Casimir, H. B. G. 1948, Proc. Kon. Ned. Akad. Wetensch., B51, 793795.

Chaboyer, B., Demarque, P., Kernan, P. J., \& Krauss, L. M. 1996, Science, 271(5251), 957-961.

Chen, W. \& Wu, Y.-S. 1990, Phys. Rev. D, 41(2), 695-698.

Einstein, A. 1917, “Kosmologische Betrachtungen zur allgemeinen Relativitaetstheorie" (Cosmological Considerations in the General Theory of Relativity)", Sitzungsberichte der Königlich Preussischen Akademie der Wissenschaften Berlin, 142-152. (In German)

Friedmann, A. 1922, “Uber die Krummung des Raumes”, Zeitschrift fur Physik, 10, 377-386. (In German). English version - Friedmann, A. 1999, “On the Curvature of Space”, General Relativity and Gravitation, 31(12), 1991-2000.

Fujii, Y. \& Nishioka, T., 1990, Phys. Rev. D, 42(2), 361-370.

Guth, A. H. 1981, Phys. Rev. D, 23(2), 347-356.

Guth, A. H. 1997, Beam Line, 27(3), 14-2. (https://ned.ipac.caltech. edu/level5/Guth/Guth_contents.html).

Hobson, A., 2013, American Journal of Physics, 81(3), 211-223.

Lamoreaux, S. K. 1997, Physical Review Letters, 78(1), 5-8.

Lattman, E. E. 2009, European Journal of Physics, 30, L41-L42.

Mohideen U. and Anushree, R. 1998, Physical Review Letters, 81(21), 4549-4552.

Peebles, P. J. E., 2003, Reviews of Modern Physics, 75, 559-606.

Planck Collaboration, 2016, A\&A, 594, id.A13 (63pp).

Rubakov, V. A. 2000, Phys. Rev. D, 61, 061501.

Saslow, W. M. 1998, European Journal of Physics, 19, 313.

Velten, H. E. S., vom Marttens, R. F., Zimdahl, W. 2014, Eur. Phys. J. C, 74, 3160 .

Weinberg, S. 1989, Reviews of Modern Physics, 61(1), 1-23.

Wilson, C. M., Johansson, G., Pourkabirian, A., Simoen, M., Johansson, J. R., Duty, T. et al. 2011, Nature, 479, 376-379.

Yousaf, Z., Bamba, K., \& Bhatti M. Z. 2016a, Phys. Rev. D, 93, 124048.

Yousaf, Z., Bamba, K., \& Bhatti, M. Z. 2016b, Phys. Rev. D, 93, 064059.

Yousaf, Z. 2017, Eur. Phys. J. Plus, 132, 71.

Yousaf, Z. 2018, Astrophys. Space Sci., 363, 226.

Yousaf, Z. 2019a, Eur. Phys. J. Plus, 134, 245.

Yousaf, Z., Bamba, K., Bhatti, M. Z., \& Ghafoor, U., 2019b, Phys. Rev. D, 100, 024062.

Zlatev, I., Wang, L., and Steinhardt, P. J., 1999, Physical Review Letters, 82(5), 896-899. 